\title{
E23 COUPLING FINITE VOLUME AND BOUNDARY ELEMENT METHODS FOR FLOW MODELLING
}

L. JEANNIN, R. BASQUET, Y. DING

Institut Français du Pétrole, 1 \& 4 avenue bois Préau 92400 Rueil-Malmaison

\begin{abstract}
Flow modelling in heterogeneous media is a great challenge, especially in the presence of wells or hydraulic fractures.

The most used numerical scheme, finite volume method, allows taking heterogeneities into account. But finite volume meshes adapted to the geometry of wells or fractures cannot be built efficiently. Conversely, Boundary element method (BEM) can be used to model complex geometries in homogeneous media. The use of BEM requires working on homogeneized area and accepting to loose the information about heterogeneities.

In this paper, we propose an innovative method to solve this conflict and to use the combined advantages of both methods: we develop an approach using the coupling of finite volume scheme and boundary element method. The BEM is only used in reduced areas of complex geometries. The existence and uniqueness of the solution of our coupling scheme is mathematically proved and its performance is numerically studied.

Finally, we show how our coupling scheme can bring a solution to the problem of modelling objects of complex geometry in heterogeneous media.
\end{abstract}

\section{Introduction}

BEM has been used in well tests in reservoir engineering [5,9]. As example, Pecher and Stanislas [9] proposed a resolution of a multi-domain integral methods to simulate well tests. The main interest of the boundary integral method is its ability to describe complex geometry. So it seems to be tempted to use boundary element methods around objects of complex geometry such as advanced wells or fractures and to use more classical discrete numerical scheme far from the well. In this paper, we consider a steady-state problem. One application of such procedure is the calculus of well performance in an heterogeneous reservoir. Another application could be the homogeneization of geological objects. Moumas et al.[7] propose a method to discretize wells using integral methods.

In this paper, we deal with the problem of coupling a finite volume scheme and a boundary integral representation of an interior domain. The first part describes the simplified problem considered and presents the discretization in space. In the second part, we generalize this approach to several bounded and simply connected domains, where the solution is given by an integral representation. Part 3 is devoted to numerical tests. 


\section{The Boundary Element Method and the Interface problem}

The coupling of boundary element and mixed finite element or finite element methods has been studied in recent papers. Meddahi et al. [6] present a numerical method for solving an exterior Dirichlet problem in the plane by coupling mixed finite element with an integral representation of the solution in an exterior unbounded region. Costabel et al. [2], Mund et al. [8] use a symmetric coupling of finite element and boundary element to resolve a parabolic-elliptic interface problem.

Finite volume methods are widely used in reservoir simulation. We propose a coupling method between finite volume and an integral representation of the solution in an interior domain.

\subsection{The Interface problem}

We give a brief description of the coupling method we adopt. A proof of the existence and the uniqueness of the discrete solution is given in Annexe. Then we generalize this approach with multi-domain boundary integral method.

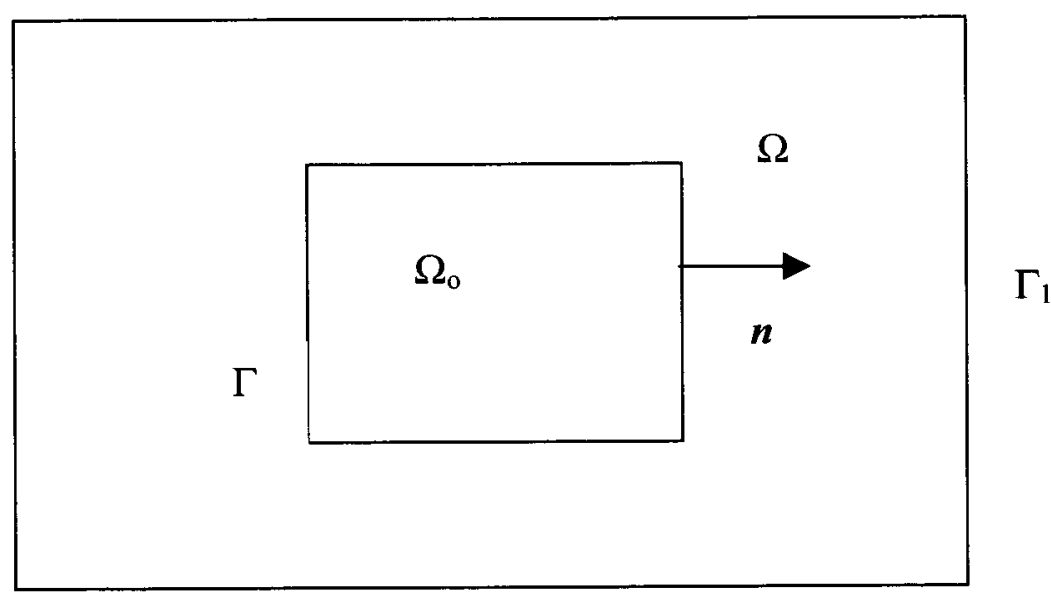

Figure 1: Geometry of the problem

Let $\Omega_{0}$ be a bounded and simply connected domain of $\mathbf{R}^{2}$ with a boundary $\Gamma$. Let $\Omega$ the region limited by $\Gamma$ and $\Gamma_{1} .\left(\Gamma_{1}\right.$ is supposed to be strictly contained in $\left.\mathbf{R}^{2}-\bar{\Omega}_{o}\right)$.

We consider the following problem $\mathrm{P} 1$ (permeability noted $k$ is supposed isotropic):

$$
\left\{\begin{array}{l}
\kappa_{o} \Delta p_{o}=0 \text { in } \Omega_{o} \\
\kappa \Delta p=0 \text { in } \Omega \\
p_{o}-p=0 \quad \text { on } \Gamma \quad \text { and } \quad \kappa_{o} \frac{\partial p_{o}}{\partial n}-\kappa \frac{\partial p}{\partial n}=0 \quad \text { on } \Gamma \\
p=p_{D} \quad \text { on } \quad \Gamma_{D} \quad \text { and } \quad \kappa \frac{\partial p}{\partial n}=h \quad \text { on } \Gamma_{N} \quad\left(\Gamma_{D} \cup \Gamma_{N}=\Gamma_{1}\right)
\end{array}\right.
$$

We suppose $\kappa=\kappa_{0}=1$

We solve the problem P1 using finite volume in $\Omega$ and an integral representation on $\Gamma$ of the solution in $\Omega_{\mathrm{o}}$. 


\section{$\underline{1.2 \text { Finite volume }}$}

A centered finite volume discretization is used in $\Omega$. Let's consider a mesh of the domain $\Omega$. the mesh is supposed to be admissible in the context of finite volume methods [3] (cartesian grid, voronoi grid...). To discretize $\Delta \mathrm{p}=0$ on each cell of the finite volume mesh, we use Green formula and a first order approximation of the pressure to evaluate the flux on each edges $\lambda$. We obtain:

On each cell $k, \quad \sum_{i} T_{i k}\left(P_{i}-P_{k}\right)=0$

$T_{i k}=\frac{l_{i k}}{d(i, k)}$

where $\mathrm{i}$ denotes the cell having a common edge with the cell $\mathrm{k}$; $l_{i k}$ is the length of the edge, and $d(i, k)$ the distance between the center of the cell $\mathrm{i}$ and the cell $\mathrm{k}$. $\mathrm{T}_{i k}$ is the transmissivity of the edge considered.

\section{$\underline{1.3 \text { Boundary Integral Method }}$}

We note in this paragraph $g$ the unknown pressure on the contour $\Gamma$ and $u . v$ the derivative of $g$ in the direction of the normal vector $\mathbf{n}=\left(\mathrm{n}_{\mathrm{x}}, \mathrm{n}_{\mathrm{y}}\right)$ defined in Figure 1 .

Let $G(x-y)=\frac{1}{2 \pi} \log (|x-y|)$ be the fundamental solution of the two dimensional Laplacian. The pressure $\mathrm{g}$ on the contour $\Gamma$ and the normal flux can be represented respectively by the singular and the hypersingular equations:

$$
\left\{\begin{array}{l}
\frac{1}{2} g(x)=-\int_{\Gamma} \frac{\partial g}{\partial n_{y}} G(x-y) d \gamma(y)+\int_{\Gamma} \frac{\partial G(x-y)}{\partial n_{y}} g(y) d \gamma(y) \\
\frac{1}{2} \frac{\partial g(x)}{\partial n_{x}}=-\int_{\Gamma} \frac{\partial g}{\partial n_{y}} \frac{\partial G(x-y)}{\partial n_{x}} d \gamma(y)+\frac{\partial}{\partial n_{x}} \int_{\Gamma} \frac{\partial G(x-y)}{\partial n_{y}} g(y) d \gamma(y)
\end{array}\right.
$$

We introduce the following boundary integral operators which associate to the function $\xi$ the following functions:

$$
\begin{aligned}
& V \xi(x)=-\int_{\Gamma} G(x-y) \xi(y) d \gamma(y) \\
& D \xi(x)=\frac{\partial}{\partial n_{x}} \int_{\Gamma} \frac{\partial G(x-y)}{\partial n_{y}} \xi(y) d \gamma(y) \\
& K \xi(x)=\int_{\Gamma} \frac{\partial G(x-y)}{\partial n_{y}} \xi(y) d \gamma(y) \\
& K^{\prime} \xi(x)=\int_{\Gamma} \frac{\partial G(x-y)}{\partial n_{x}} \xi(y) d \gamma(y)
\end{aligned}
$$

Using these definitions, the two integral formulations for pressure and normal flux can be written as follows: 
$g=V u . v+\left(\frac{I d}{2}+K\right) g$

$u . v=D g+\left(\frac{I d}{2}-K^{\prime}\right) u . v \quad$ or $\quad D g-\left(\frac{I d}{2}+K^{\prime}\right) u . v=0$

We construct a variational formulation of the singular equation associated to pressure and the hypersingular integral equation associated to normal flux. We consider the discretization of this weak formulation. The finite volume mesh built a partition $\Gamma_{\mathrm{h}}$ in $\mathrm{M}$ edges of the contour $\Gamma$. We note $S_{h}$ the set of piecewise constant function on $\Gamma_{h}$ and $G_{h}$ the set of piecewise linear function on $\Gamma_{\mathrm{h}}$. The pressure $\mathrm{g}$ is supposed to be linear on each edge of the contour and is decomposed on the basis $\left(G_{h, i}\right)_{i=1, M}$ of $G_{h}$, u.v is supposed to be constant on each edge and is decomposed on the basis $\left(Q_{h, i}\right)_{i=1, M}$ of $S_{h}$.

$$
\begin{aligned}
& g=\sum_{i=1, M} g_{i} G_{h, i} \\
& u . v=\sum_{i=1, M} q_{i} Q_{h, i}
\end{aligned}
$$

We test the singular boundary integral equation against the function of $S_{h}$ (We multiply by a test function of $S_{h}$ and integrate on $\Gamma$ ) and the hypersingular boundary integral equation against the function of $G_{h}$.

$$
\begin{aligned}
& \left\langle g \mid Q_{h, i}\right\rangle=\left\langle v u . v \mid Q_{h, i}\right\rangle+\left\langle\left(\frac{I d}{2}+K\right) g \mid Q_{h, i}\right\rangle \quad \forall \quad Q_{h, i} \in S_{h} \\
& \left\langle u . v \mid G_{h, i}\right\rangle=\left\langle D g \mid G_{h, i}\right\rangle+\left\langle\left(\frac{I d}{2}-K^{\prime}\right) u . v \mid G_{h, i}\right\rangle \quad \forall \quad G_{h, i} \in G_{h}
\end{aligned}
$$

with $\langle g \mid \mu\rangle=\int_{\Gamma} g(x) \mu(x) d x$

The main advantages of a symmetric variational formulation are :

- the hypersingular operator $\mathrm{D}$ is regularized by the integration of the weak formulation and can be more easily evaluated $[1,4]$,

- the linear system of the interface problem coupling finite volume and boundary element methods can be inverted (see annexe)

Boundary integral operators are calculated using analytical integration or gaussian quadrature rules when necessary.

Note the following properties of the boundary integral matrices: $\mathrm{D}_{\mathrm{ij}}$ is a positive semi-defined matrix (ker $\mathrm{D}=\mathbf{R}) \cdot \mathrm{V}_{\mathrm{ij}}$ is a positive defined matrix or can be considered as a positive defined matrix (this can always be obtained by scaling - see $[2,8]) .(\mathrm{Id} / 2+\mathrm{K})_{\mathrm{ij}}$ and $\left(\mathrm{Id} / 2+\mathrm{K}^{\prime}\right)_{\mathrm{ij}}$ are adjoint matrices.

$$
\left\langle\left(\frac{I d}{2}+K^{\prime}\right) Q_{h, j} \mid G_{h, i}\right\rangle=\left\langle\left(\frac{I d}{2}+K\right) G_{h, j} \mid Q_{h, i}\right\rangle
$$

\subsection{Coupling of Finite Volume with Boundary Integral Method}

The singular boundary integral equation defined an interface pressure $P_{m}$ on each edge of the contour $\Gamma$. 


$$
P_{h, i}=\left\langle V u_{1} \cdot v \mid Q_{h, i}\right\rangle+\left\langle\left(\frac{I d}{2}+K\right) g_{1} \mid Q_{h, i}\right\rangle \quad \forall \quad Q_{h, i} \in S_{\Gamma_{h}}
$$

To solve the problem P1, we define three types of discrete unknowns:

$-\mathrm{P}_{\mathrm{k}}$ the finite volume pressure on each cell $\mathrm{k}$ of the finite volume mesh,

$-P_{h, j}$ the interface pressure on each edge of the contour $\Gamma_{h}$,

- $g$ the unknown pressure of the integral method defined on each vertex of the contour $\Gamma_{h}$.

The linear system is composed of $M$ (number of cells of the finite volume mesh) $+2 \mathrm{~N}(\mathrm{~N}$ vertices and $\mathrm{N}$ edges of the contour $\Gamma$ ) equations:

-the first set of equations is the resolution of $\Delta \mathrm{p}=0$ on each cell of $\mathrm{M}$ cells of the finite volume mesh.

-the second set of equations is the singular boundary integral equation on $\Gamma$ which defines an interface pressure on each edge ( $\mathrm{N}$ equations).

-the third set of equations used is the hypersingular boundary integral equation written on each vertex of the contour $\Gamma$ ( $\mathrm{N}$ equations).

The continuity of pressure and flux on the contour $\Gamma$ by expressing the flux on each edge in the direction of $\mathbf{n}$ as:

$F=-T_{\text {bord }}\left(P_{h, i}-P_{k}\right)$

$\mathrm{T}_{\text {bord }}$ is the transmissivity of the edge considered in the finite volume mesh.

Using the boundary conditions expressed in 1.1 , the linear system is inversible. So, we solve a finite volume/BEM method coupling problem without using a mesh of $\Omega_{\mathrm{o}}$.

\section{Coupling of two domain integral method}

We generalize the precedent method with multi-domain described by method integrals and embedded in a finite volume mesh.

Consider the domain $\Omega_{0}$ defined in figure 1 and a partition of this domain in two simply connected domain $\Omega_{1}$ and $\Omega_{2}$ (bounded respectively by $\Gamma_{1}$ and $\Gamma_{2}$ ). We impose the continuity of the pressure and the flux across the intersection of the contour $\Gamma_{1}$ and $\Gamma_{2}$.

$$
\left\{\begin{array}{l}
\left\langle u_{1} \cdot v \mid Q_{h}\right\rangle=-\left\langle u_{2} \cdot v \mid Q_{h}\right\rangle \quad \forall \quad Q_{h} \in S_{\Gamma_{1}, h} \cap S_{\Gamma_{2}, h} \\
\left\langle v u_{1} \cdot v+\left(\frac{I d}{2}+K\right) g_{1} \mid Q_{h}\right\rangle=\left\langle V u_{2} \cdot v+\left(\frac{I d}{2}+K\right) g_{2} \mid Q_{h}\right\rangle \quad \forall \quad Q_{h} \in S_{\Gamma_{1}, h} \cap S_{\Gamma_{2, h}}
\end{array}\right.
$$

This approach allows to deal with different domain solved by integral methods with different permeabilities (constant on each domain).

\section{Numerical tests}

- We firstly test the method on heterogeneous media, for which we have analytical solution (we use stratified media with heterogeneities described by domains solved using boundary or finite volume methods). This method is a good candidate for calculus of homogeneization on objects of complex geometry. 

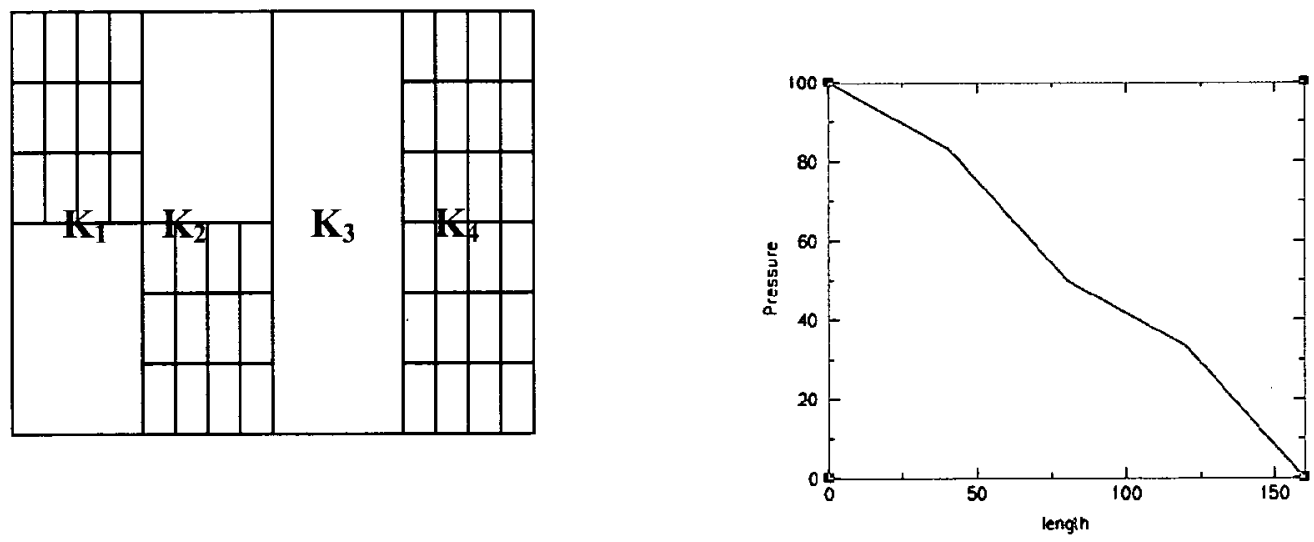

Figure 2: Areas with mesh correspond to the use of finite volume method; in the other areas boundary element method is used. Pressure and flux are correctly transmitted through the interfaces $\left(\mathrm{K}_{3}=\mathrm{K}_{1}=2 \mathrm{~K}_{2}=2 \mathrm{~K}_{4}\right)$.

- The figure 3. shows two tests of the coupling method, one with a complex interface coupling two integral domains and the other coupling finite volume and boundary integral methods in an heterogeneous medium.

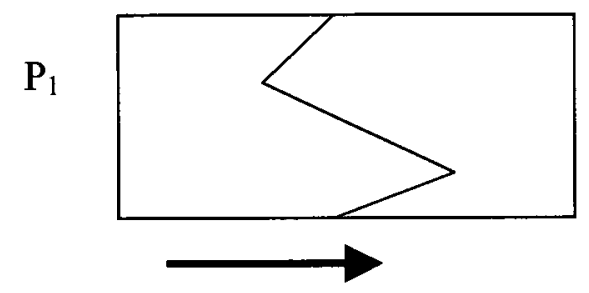

Figure 3.a: With the boundary conditions of the permeameter, the pressure at the interface is correctly transmitted across a complex interface (linearity is conserved - we use in this case a great number of quadrature points to evaluate the integrals)

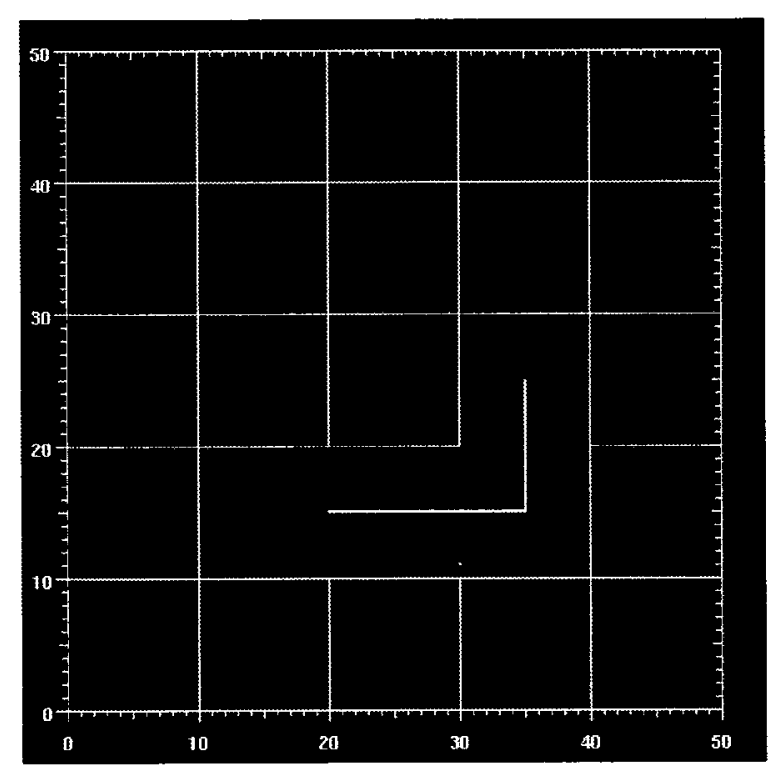

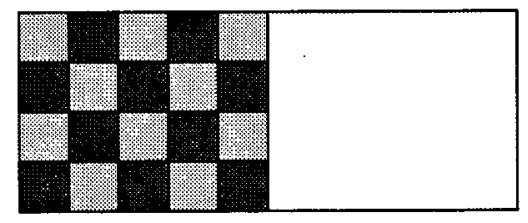

Figure 3.b: We compare the pressure field $P_{V F}$ of a regular finite volume mesh with the pressure field $\mathrm{P}_{\mathrm{MI}}$ of the coupling problem illustrated by the figure 3.b for the same permeability field. (Each color corresponds to a value of permeability).

Sup $\left|\mathrm{P}_{\mathrm{VF}}-\mathrm{P}_{\mathrm{MI}}\right| / \mathrm{P}_{\mathrm{VF}}<0.005$ at the interface

Same boundary conditions as in $3 . \mathrm{a}$

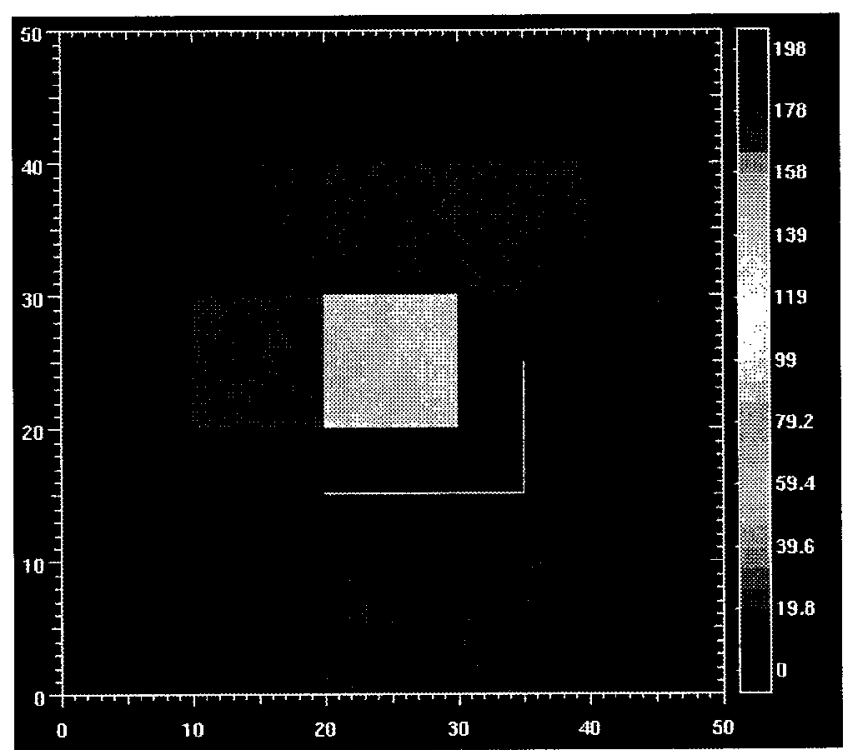

Figure 4: Pressure map - The pressure imposed at the fracture is 200 bars and the pressure at the exterior boundary is 100 bars (here the medium is supposed homogeneous). 
- The third test illustrates one application of the coupling method. A well is connected with a fracture and the fracture is described as a constant pressure line source at the interface of two domains solved with boundary integral method and embedded in finite volume mesh.

\section{Conclusion}

In this paper, a new approach is proposed to model geological objects of complex geometry. We combine the advantages of finite volume and boundary element methods. The main advantages of such an approach will be the use of boundary element method only in areas of complex geometry.

We develop a numerical method to couple finite volume and boundary element methods. The existence and uniqueness of the discrete solution has been obtained. A generalization to not simply connected domains solved with integral methods will be studied in a forthcoming paper.

\section{References}

[1] Bonnet M. - Equations Intégrales et éléménts frontières, Applications en mécanique des solides et des fluides, CNRS Editions / Eyrolles, 1995

[2] Costabel M., Ervin V.J., Stephan E.P., Symmetric coupling of finite elements and boundary elements for a parabolic-elliptic interface problem, Quaterly of Applied Mathematics, vol XLVIII, pp265-279, 1990

[3] Eymard R., Gallouet T, Herbin R., Finite Volume methods, handbook of Numerical Analysis, eds P.G. Ciarlet and Lions J.L.

[4] Giroire J - Cours de DEA de l'université de Nantes - 1992

[5] Kikani J. and Horne R.N., Pressure-Transient Analysis of Arbitrarily Shaped Reservoirs with the boundary element method, SPE 18159, p53-60, 1998

[6] Meddahi S., Valdes J., Menendez O., Perez P., On the coupling of boundary integral and mixed finite element methods, Journal Comput. Appl. Math., 69(1), 127-141, 1996

[7] Moumas V., Ding D., Giroire J., Evaluation of the performance of complex wells by boundary integral methods, ECMOR VIII, 2002

[8] Mund P, Sprehan E. P. , Adaptive Coupling and fast solution of FEM-BEM Equations for Parabolic-Elliptic Interface Problems, Mathematical A ethods in the applied Sciences, Vol. 20, 403-423, 1997

[9] Pecher R. and Stanislav J.F., Boundary element techniques in petroleum reservoir simulation, Journal of Petroleum Engineering, 17, p353-366, 1997

\section{Annexe}

Let's consider the problem P2: 


$$
\left\{\begin{array}{l}
\kappa_{o} \Delta p_{o}=0 \text { in } \Omega_{o} \\
\kappa \Delta p=0 \text { in } \Omega \\
p_{o}-p=0 \quad \text { on } \Gamma \quad \text { and } \kappa_{o} \frac{\partial p_{o}}{\partial n}-\kappa \frac{\partial p}{\partial n}=0 \quad \text { on } \Gamma \\
p=0 \quad \text { on } \quad \Gamma_{D} \quad \text { and } \kappa \frac{\partial p}{\partial n}=0 \quad \text { on } \Gamma_{N} \quad\left(\Gamma_{D} \cup \Gamma_{N}=\Gamma_{1}\right)
\end{array}\right.
$$

We suppose $\kappa=\kappa_{0}=1$

If two solutions verify problem $\mathrm{P} 1$, then their difference verifies problem $\mathrm{P} 2$. We show that the linear system corresponding to the resolution of the problem P2 has a unique solution which is (0).

Multiplying the $\mathrm{k}^{\text {th }}$ line of the linear system by $-P_{k}$ for the $\mathrm{M}$ first lines and adding them, we obtain:

$$
\sum_{\delta} T_{i k}\left(P_{i}-P_{k}\right)^{2}+\sum_{\delta \in \Gamma_{h}} P_{k} F_{b o r d, \delta}+\sum_{\delta \in \Gamma_{D}} T_{\delta} P_{k}^{2}=0
$$

Multiplying the $\mathrm{N}$ following lines by $Q_{\text {bord }, \delta}=F_{\text {bord }, \delta} / 1_{\delta}$ adding them, we obtain:

$$
\sum_{\delta \in \Gamma_{h}}-\left\langle P_{b o r d, \delta} \mid Q_{b o r d, \delta}\right\rangle+\sum_{\delta \in \Gamma_{h}}\left\langle V Q \mid Q_{b o r d, \delta}\right\rangle+\sum_{\delta \in \Gamma_{h}}\left\langle\left(\frac{I d}{2}+K\right) g \mid Q_{b o r d, \delta}\right\rangle=0
$$

Multiplying the $\mathrm{N}$ last lines by $\mathrm{g}_{\mathrm{v}}$ and adding them, we obtain:

$$
\sum_{v \in \Gamma_{h}}\left\langle D g \mid g_{v}\right\rangle-\sum_{v \in \Gamma_{h}}\left\langle\left(\frac{I d}{2}+K^{\prime}\right) Q \mid g_{v}\right\rangle=0
$$

Adding this three equalities and taking account that $(\operatorname{Id} / 2+\mathrm{K})$ and $\left(\operatorname{Id} / 2+\mathrm{K}^{\prime}\right)$ are adjoint operators, we obtain:

$$
\begin{gathered}
\sum_{\delta} T_{i k}\left(P_{i}-P_{k}\right)^{2}+\sum_{\delta \in \Gamma_{h}} P_{k} F_{b o r d, \delta}+\sum_{\delta \in \Gamma_{D}} T_{\delta} P_{k}^{2}-\sum_{\delta \in \Gamma_{h}} P_{b o r d, \delta} F_{b o r d, \delta}+\langle V Q \mid Q\rangle \\
+\langle D g \mid g\rangle=0
\end{gathered}
$$

or

$$
\sum_{\delta} T_{i k}\left(P_{i}-P_{k}\right)^{2}+\sum_{\delta \in \Gamma_{D}} T_{\delta} P_{k}^{2}+\sum_{\delta \in \Gamma_{h}} T_{b o r d, \delta}\left(P_{k}-P_{b o r d, \delta}\right)^{2}+\langle V Q \mid Q\rangle+\langle D g \mid g\rangle=0
$$

We deduced from this equality that :

$\forall k \quad P_{k}=0$

$\forall \delta \quad P_{b o r d, \delta}=0$

$\forall \delta \quad Q_{\text {bord }, \delta}=0$

Moreover, $\operatorname{ker} \mathrm{D}=\mathbf{R} \cdot \mathrm{g}$ is a constant $\lambda$ function on $\Gamma$.

But for the integral representation of an interior problem on a simply connected domain, $\operatorname{ker}\left(\frac{i d}{2}+K\right) \cap \operatorname{ker} D=\{0\}$

So $g_{v}=0$ on $\Gamma_{h}$

$(0)$ is a unique solution of the linear system. 